\title{
Hybrid arch frozen elephant trunk repair: evidence from the Canadian Thoracic Aortic Collaborative
}

\author{
Maral Ouzounian ${ }^{1}$, Ali Hage ${ }^{2}$, Jennifer Chung ${ }^{1}$, Louis-Mathieu Stevens ${ }^{3}$, Ismail El-Hamamsy ${ }^{4}$, \\ Vincent Chauvette ${ }^{3}$, Francois Dagenais ${ }^{5}$, Andreanne Cartier ${ }^{5}$, Mark Peterson ${ }^{1}$, Alana Harrington ${ }^{1}$, \\ Munir Boodhwani ${ }^{6}$, Ming Guo ${ }^{6}$, John Bozinovski ${ }^{7}$, Stephanie Fox ${ }^{2}$, Linrui Guo ${ }^{2}$, Michael W. A. Chu ${ }^{2}$; on \\ behalf of the Canadian Thoracic Aortic Collaborative
}

${ }^{1}$ Division of Cardiac Surgery, Department of Surgery, University of Toronto, Toronto, ON, Canada; ${ }^{2}$ Division of Cardiac Surgery, Department of Surgery, Western University, London, ON, Canada; ${ }^{3}$ Division of Cardiac Surgery, Department of Surgery, University of Montreal, Montreal, QC, Canada; ${ }^{4}$ Department of Cardiovascular Surgery, Mount Sinai Hospital, Icahn School of Medicjne at Mount Sinai, New York, NY, USA; ${ }^{5}$ Division of Cardiac Surgery, Department of Surgery, Laval University, Quebec City, QC, Canada; ${ }^{6}$ Division of Cardiac Surgery, Department of Surgery, University of Ottawa, Ottawa, ON, Canada; ${ }^{7}$ Division of Cardiac Surgery, Ohio State University Wexner Medical Center, Columbus, OH, USA Correspondence to: Maral Ouzounian, MD, PhD, FRCSC. Associate Professor of Surgery, Peter Munk Cardiac Centre, University of Toronto, 200 Elizabeth St. 4N-464, Toronto, ON M5G 2C4, Canada. Email: Maral.Ouzounian@uhn.ca.

Background: The frozen elephant trunk (FET) technique has become an increasingly popular strategy for aortic reconstruction in the setting of extensive thoracic aortic aneurysms or dissections. The objective of this study is to report on the Canadian experience with the FET technique in both the elective and emergent settings.

Methods: A total of 167 consecutive patients (mean age $65 \pm 13$ years, $30 \%$ female, $25 \%$ re-operation) underwent elective $(70 \%)$ and non-elective $(30 \%)$ aortic arch reconstruction with the FET technique between May 2008 and October 2019 in six centers of the Canadian Thoracic Aortic Collaborative (CTAC). In-hospital clinical endpoints and early imaging endpoints were prospectively collected and analyzed.

Results: All 167 patients underwent successful FET implantation. In-hospital mortality occurred in 14 patients $(8 \%)$, stroke occurred in 22 patients (13\%) and temporary and permanent spinal cord ischemia (SCI) occurred in $6(3.6 \%)$ and $3(1.8 \%)$ patients, respectively. Prolonged mechanical ventilation was required in 35 patients $(21 \%)$, renal failure requiring dialysis in 14 patients $(8 \%)$ and atrial fibrillation in 59 patients (36\%). The median hospital and intensive care unit (ICU) lengths of stay were 3 [interquartile range (IQR): 1, 6] and 10 (IQR: 7, 17) days, respectively. The rate of type $1 \mathrm{~A}$ endoleak was $3.6 \%$, with the lowest rate in patients who underwent a total arch replacement with a hybrid FET graft $(0 \%)$ and the highest among patients who had a hemiarch with antegrade thoracic endovascular aortic repair (TEVAR) deployment (25\%). The rate of other types of endoleak and stent complications was comparatively low.

Conclusions: The early CTAC experience with the FET operation demonstrates technical feasibility and good early clinical outcomes in elective and emergent patients. Further analysis is required to explore variations in technique and their potential impact on early and late outcomes.

Keywords: Hybrid arch repair; frozen elephant trunk (FET); aortic arch

Submitted Jan 18, 2020. Accepted for publication May 05, 2020.

doi: $10.21037 /$ acs-2020-fet-22

View this article at: http://dx.doi.org/10.21037/acs-2020-fet-22 


\section{Introduction}

The frozen elephant trunk (FET) technique for reconstruction of the aortic arch has become an established strategy to address extensive thoracic aneurysms or dissections through a median sternotomy (1-4). Compared to a conventional elephant trunk, the FET has several potential benefits: (I) it permits single stage repair for extensive aneurysmal disease limited to the thoracic aorta; (II) delays or avoids a second stage repair in patients with chronic dissections; (III) reduces the likelihood of kinking, obstruction, or accordioning compared to a soft conventional elephant trunk and facilitates distal endovascular repair; (IV) in the setting of acute DeBakey I aortic dissection, a FET may be particularly advantageous in improving distal malperfusion and promoting favorable early and late distal aortic remodeling. Given these potential advantages, there has been rapid uptake of the FET technique for patients with complex thoracic aneurysms and dissections.

The objective of this study is to report on the Canadian experience with the FET technique in both the elective and emergent setting. Several devices are available within the repertoire of FET arch repair, each with perceived advantages and disadvantages. We aimed to evaluate the contemporary in-hospital outcomes and adverse events of aortic arch reconstruction with FET using a wide variety of devices and techniques.

\section{Methods}

\section{Study population}

The Canadian Thoracic Aortic Collaborative (CTAC) is a national collaborative of cardiac surgeons with expertise in complex aortic repair across Canada who retrospectively compiled a comprehensive registry of consecutive patients undergoing thoracic aortic surgery with circulatory arrest. Circulatory arrest cases for thoracoabdominal aortic repair, or for those not involving aortic repair (i.e., congenital cases, tumor removal, etc.) were excluded. Each center obtained local ethics approval from their respective institutional review boards, and individual informed consent was waived at all centers.

For the present study, all consecutive patients from six institutions who underwent arch repair with a FET technique between May 2008 and October 2019 were analyzed. FET repairs in this study included a variety of techniques and devices including hybrid arch grafts such as the Thoraflex Hybrid (Vascutek, Glasgow, Scotland), Evita OPEN PLUS (Jotec, Hechingen, Germany/CryoLife, Kennesaw, GA, USA), and Cook Hybrid (Cook Medical, Bloomington, IN, USA) graft (Figure 1); off-label antegrade deployment of commercially available thoracic endovascular aortic repair (TEVAR) (Figure 2); and home-made FET grafts. Elective and emergent cases were included, as were cases with concomitant surgery.

\section{Statistical methods}

Continuous variables were expressed as mean \pm standard deviation or median [interquartile range (IQR)], and categorical variables were expressed as frequencies (\%). Statistical analysis was conducted using Stata version 16.0 (StataCorp LLC, College Station, TX, USA).

\section{Surgical technique}

Although there was some variability in technique, general operative conduct was similar at all centers. The right axillary artery through a Dacron side-graft was the most common route for arterial cannulation. After systemic heparinization, a guide-wire was inserted through a femoral sheath and guided with echocardiography and/ or fluoroscopy into the distal aortic arch. In cases of dissection, this was confirmed by imaging to be in the true lumen. Any aortic root work or concomitant procedures were performed during cooling to moderate hypothermia. During circulatory arrest, cerebral protection was most commonly established via unilateral antegrade cerebral perfusion (ACP) with near-infrared spectroscopy for monitoring. Most cases were performed with a hybrid graft where the FET collar was sewn at zone 2. The FET grafts were deployed over a guidewire during circulatory arrest. The head vessels were either re-implanted onto the individual hybrid graft branches or sewn prior to circulatory arrest using a bi- or trifurcated graft. Several variations were employed for dealing with the left subclavian artery, which have previously been described (5). Systemic reperfusion was restored via a side-limb or cannula in the arch graft. The arch graft was connected to the root graft or ascending aortic graft to allow cardiac reperfusion. The FET positioning and deployment was assessed on transesophageal echocardiography and/or fluoroscopy ensuring that the stented portion of the grafts were well 
A



B

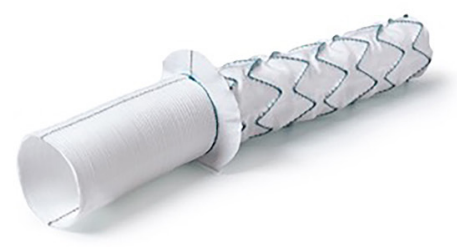

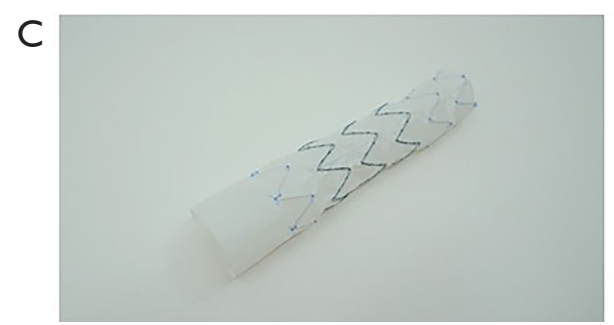

Figure 1 Illustration of the Thoraflex Hybrid Graft (Vascutek, Glasgow, Scotland) (A), Evita OPEN PLUS (Jotec, Hechingen, Germany/ CryoLife, Kennesaw, GA, USA) (B), and Cook custom made endovascular hybrid arch graft (Cook Medical, Bloomington, IN, USA) (C).

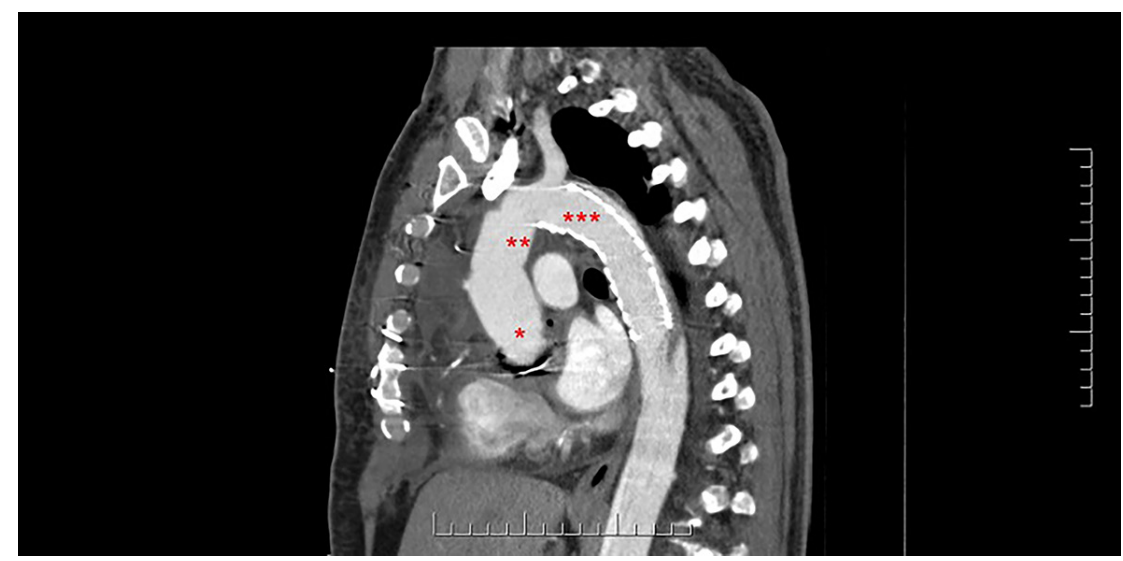

Figure 2 Sagittal view of CT of a patient who had a Bentall procedure $\left(^{*}\right)+$ hemiarch replacement $\left.{ }^{* *}\right)+$ antegrade Gore TAG graft $\left({ }^{* * *}\right)$ for a type A dissection with mal-perfusion. CT, computed tomography.

expanded with no kinks or turbulent flow.

\section{Results}

\section{Baseline characteristics}

Table 1 summarizes the baseline characteristics of 167 patients who received a FET at participating CTAC institutions. Mean age was $65 \pm 13$ years; $30 \%$ were female; $81 \%$ were hypertensive; and $4 \%$ had a suspected or confirmed connective tissue disorder. Among the 167 patients, $14 \%$ had a history of cerebrovascular disease with previous stroke or transient ischemic attack; and $25 \%$ had a previous cardiac operation via sternotomy. In this cohort, 15 patients $(9 \%)$ underwent urgent repair, 33 patients (20\%) emergent repair, and $3(2 \%)$ salvage repair. With regards to aortic pathology, $6 \%$ of patients presented with aortic rupture and $37 \%$ with aortic dissection. The mean maximum aortic diameter was $55 \pm 16 \mathrm{~mm}$. 


\begin{tabular}{|c|c|}
\hline Clinical characteristics & Result ( $n=167)$ \\
\hline \multicolumn{2}{|l|}{ Patient characteristics } \\
\hline Age (years), mean $\pm S D$ & $65 \pm 13$ \\
\hline Female, n (\%) & $50(29.9)$ \\
\hline Hypertension, n (\%) & $136(81.4)$ \\
\hline Connective tissue disorder, $\mathrm{n}(\%)$ & $7(4.2)$ \\
\hline Diabetes, n (\%) & $22(13.2)$ \\
\hline Dyslipidemia, n (\%) & $87(52.1)$ \\
\hline Renal failure, n (\%) & $11(6.6)$ \\
\hline Cerebrovascular disease, n (\%) & $18(14.2)$ \\
\hline Peripheral vascular disease, $\mathrm{n}(\%)$ & $27(16.2)$ \\
\hline Smoker, n (\%) & $92(55.4)$ \\
\hline Chronic obstructive pulmonary disease, $\mathrm{n}(\%)$ & $23(13.8)$ \\
\hline Prior cardiac surgery, n (\%) & $42(25.2)$ \\
\hline Atrial fibrillation, $\mathrm{n}(\%)$ & $16(9.8)$ \\
\hline Coronary artery disease, $\mathrm{n}(\%)$ & $32(19.2)$ \\
\hline \multicolumn{2}{|l|}{ Anatomy } \\
\hline Body surface area $\left(\mathrm{m}^{2}\right)$, mean $\pm S D$ & $1.9 \pm 0.3$ \\
\hline Maximum aortic diameter $(\mathrm{mm})$, mean $\pm \mathrm{SD}$ & $55 \pm 16$ \\
\hline $\begin{array}{l}\text { Maximum indexed aortic diameter }\left(\mathrm{mm} / \mathrm{m}^{2}\right) \\
\text { mean } \pm \mathrm{SD}\end{array}$ & $38 \pm 16$ \\
\hline Aortic stenosis, n (\%) & $27(16.7)$ \\
\hline Aortic insufficiency, n (\%) & $71(42.5)$ \\
\hline \multicolumn{2}{|l|}{ Presentation, $\mathrm{n}(\%)$} \\
\hline Dissection & $62(37.1)$ \\
\hline Rupture & $10(6.0)$ \\
\hline \multicolumn{2}{|l|}{ Urgency status, n (\%) } \\
\hline Elective & $116(69.5)$ \\
\hline Urgent & $15(9.0)$ \\
\hline Emergent ( $<6$ hours) & $33(19.8)$ \\
\hline Salvage & $3(1.8)$ \\
\hline
\end{tabular}

\section{Intra-operative details}

Table 2 summarizes the intra-operative details of patients who received a FET. The majority of patients $(88 \%)$ had a total arch replacement and $83 \%$ had a concomitant head or neck vessel surgery. Concomitant procedures were performed in most patients, including aortic valve replacement $(14 \%)$, coronary artery bypass grafting (19\%), Bentall procedure (14\%), valve-sparing aortic root reconstruction $(5 \%)$, mitral valve surgery (3\%) and Ross procedure $(0.6 \%)$.

With regards to the perfusion details, $90 \%$ of patients received $\mathrm{ACP}$ and $4 \%$ of patients received retrograde cerebral perfusion as adjuncts during the FET repair. The median hypothermic circulatory arrest time, median [IQR] was 36 [27, 52] minutes; the lowest temperature, median [IQR] was $22\left[20,25{ }^{\circ} \mathrm{C}\right.$; and the median cerebral ischemic time, median $[\mathrm{IQR}]$ was $0[0,5]$ minutes. The majority of patients in this cohort $(81 \%)$ required transfusion of $\geq 1$ blood product perioperatively. Patients received a median [IQR] of $2[0,4]$ unit of packed red blood cells (pRBC); a median [IQR] of $2[0,4]$ units of fresh frozen plasma (FFP); and a median [IQR $]$ of $3[0,10]$ units of platelets.

\section{Early (in-hospital) clinical outcomes}

Fourteen patients (8\%) died after surgery and 22 patients (13\%) suffered a post-operative stroke. Of the latter, 2 patients had pre-operative transient ischemic attack and 2 patients had pre-operative stroke. Nine patients $(5 \%)$ experienced spinal cord ischemia (SCI) following FET repair, 6 of which were temporary $(3.6 \%)$ and 3 of which were permanent (1.8\%). Among elective patients, inhospital mortality was $3.5 \%$ and stroke was $12 \%$. Other outcomes of interest are summarized in Table 3 and included cardiac reoperation (7\%); prolonged ventilation (>40 hours) (21\%); dialysis dependent renal failure (8\%); and septicemia (8\%). Delirium was common and experienced by $25 \%$ of patients. The median intensive care unit (ICU) length of stay $[\mathrm{IQR}]$ was $3[1,6]$ days, and the median hospital length of stay [IQR] was 10 [7, 17] days. There was no association between length of FET and SCI (un-adjusted OR: 1.01, 95\% CI: 0.99-1.00, P=0.23). In an un-adjusted explanatory analysis looking at the relationship between center-volumes and outcomes, we defined a "high-volume center" as a center with more than 30 FET cases; consequently, three centers were labelled as "high-volume" and three centers were labelled as "low-volume". There was no significant association between center-volumes and outcomes for death (9.6\% for high-volume vs. $4.6 \%$ for low-volume, $\mathrm{P}=0.3$ ), stroke $(11.0 \%$ vs. $18.2 \%, \mathrm{P}=0.2)$ and SCI $(5.2 \%$ vs. $4.6 \%$, $\mathrm{P}=0.9)$. This explanatory analysis was limited by the small sample size and the infrequency of the complications. 


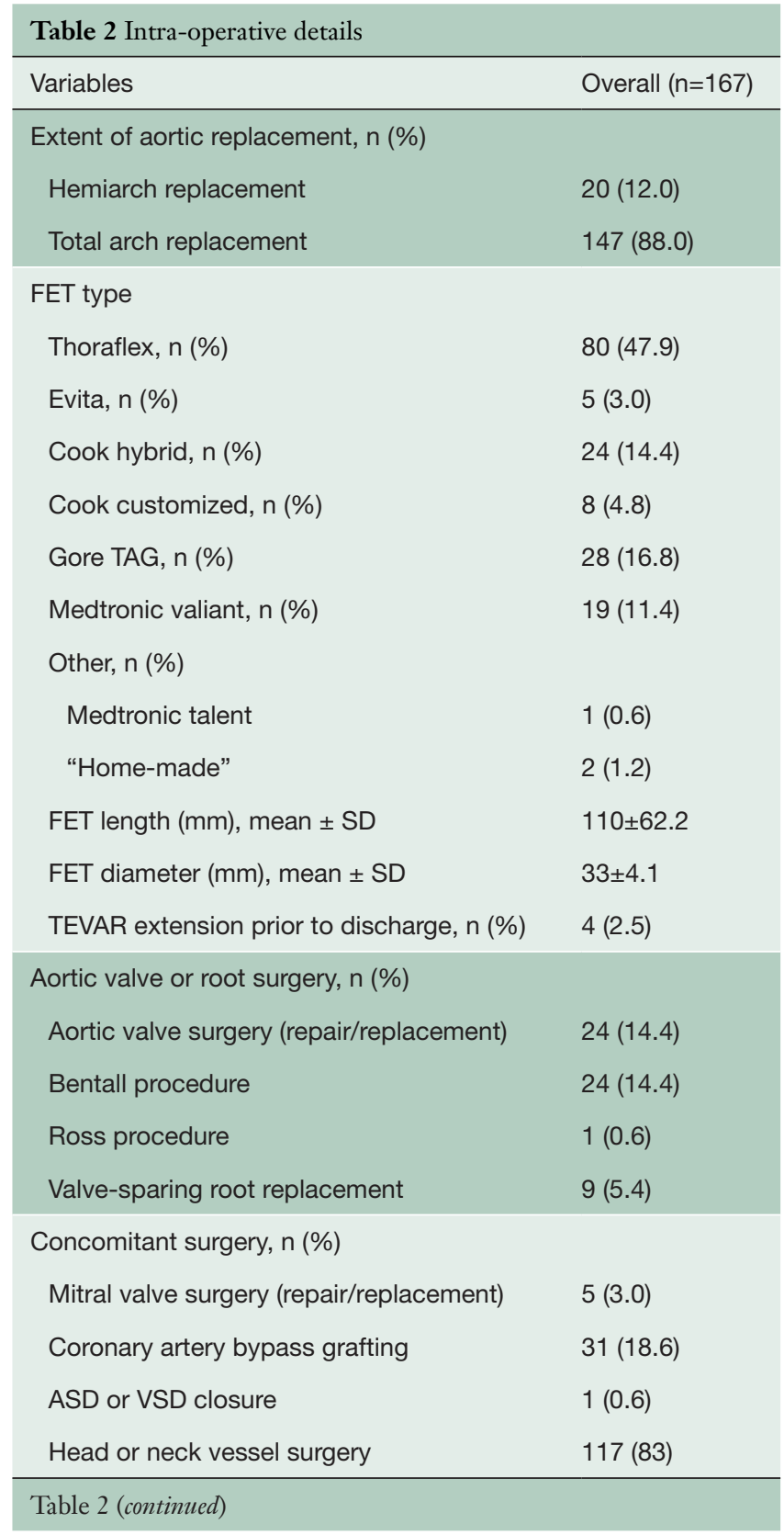

\section{Early imaging results}

When we examined the early imaging endpoints, the rate of type $1 \mathrm{~A}$ endoleak in the overall cohort was $3.6 \%$, with the lowest rate in patients who underwent a total arch replacement with a hybrid FET graft $(0 \%)$ and the highest among patients who had a hemiarch with antegrade TEVAR deployment (25\%). The rate of unplanned type $1 \mathrm{~B}$ endoleak was $1 \%$ and the rate of type 2 endoleak was $7 \%$. Type $3(0 \%)$

\begin{tabular}{|c|c|}
\hline Variables & Overall $(n=167)$ \\
\hline \multicolumn{2}{|l|}{ Perfusion } \\
\hline $\begin{array}{l}\text { Cardiopulmonary bypass time (min), median } \\
\text { [IQR] }\end{array}$ & $200[165,242]$ \\
\hline Crossclamp time (min), median [IQR] & $107[73,149]$ \\
\hline $\begin{array}{l}\text { Hypothermic circulatory arrest time (min), } \\
\text { median }[\mathrm{IQR}]\end{array}$ & $36[27,52]$ \\
\hline Lowest temperature $\left({ }^{\circ} \mathrm{C}\right)$, median $[\mathrm{IQR}]$ & $22[20,25]$ \\
\hline ACP, n (\%) & $150(89.8)$ \\
\hline Retrograde cerebral perfusion, $\mathrm{n}(\%)$ & $7(4.2)$ \\
\hline Cerebral perfusion time (min), median [IQR] & $38[25,69]$ \\
\hline Cerebral ischemia time (min), median [IQR] & $0[0,5]$ \\
\hline \multicolumn{2}{|l|}{ Transfusion } \\
\hline Any blood product transfusion, $\mathrm{n}(\%)$ & $136(81.4)$ \\
\hline $\begin{array}{l}\text { Units of pRBC in transfused patients, } \\
\text { median }[\mathrm{IQR}]\end{array}$ & $2[0,4]$ \\
\hline $\begin{array}{l}\text { Units of FFP in transfused patients, median } \\
{[\text { IQR] }}\end{array}$ & $2[0,4]$ \\
\hline $\begin{array}{l}\text { Units of platelet in transfused patients, } \\
\text { median }[\mathrm{IQR}]\end{array}$ & $3[0,10]$ \\
\hline \multicolumn{2}{|c|}{$\begin{array}{l}\text { FET, frozen elephant trunk; TEVAR, thoracic endovascular aortic } \\
\text { repair; ASD, atrial septal defect; VSD, ventricular septal defect; } \\
\text { IQR, interquartile range; ACP, antegrade cerebral perfusion; } \\
\text { pRBC, packed red blood cells; FFP, fresh frozen plasma. }\end{array}$} \\
\hline
\end{tabular}

and $4(0.6 \%)$ endoleaks were uncommon. There was no incidence of new distal aortic dissection or rupture. There was no incidence of stent migration and only one patient had a stent graft-induced new entry tear in the distal aorta.

\section{Discussion}

This study describes the inaugural Canadian experience with the FET technique with a variety of surgical approaches and devices, in both the elective and emergent setting. We observed acceptable early results with relatively low complication rates and good early clinical and imaging outcomes.

The surgical armamentarium for arch repair has expanded to include a wide variety of techniques, each with their own advantages and disadvantages. We will briefly discuss the two most common types of FET repair observed 


\begin{tabular}{|c|c|}
\hline Variables & Overall $(n=167)$ \\
\hline In-hospital mortality, n (\%) & $14(8.4)$ \\
\hline \multicolumn{2}{|l|}{ Neurological injury, n (\%) } \\
\hline Stroke & $22(13.2)$ \\
\hline Temporary neurological dysfunction & $5(3.0)$ \\
\hline Delirium & $42(25.3)$ \\
\hline Temporary SCI & $6(3.6)$ \\
\hline Permanent SCl & $3(1.8)$ \\
\hline Cardiac reoperation, $\mathrm{n}(\%)$ & $12(7.2)$ \\
\hline Need for intra-aortic balloon pump, n (\%) & $1(0.6)$ \\
\hline Prolonged ventilation (>40 h), n (\%) & $35(21.0)$ \\
\hline Atrial fibrillation, $\mathrm{n}(\%)$ & $59(35.8)$ \\
\hline Postoperative myocardial infarction, n (\%) & $2(1.3)$ \\
\hline Cardiac arrest, n (\%) & $8(4.8)$ \\
\hline Dialysis dependent renal failure, $\mathrm{n}(\%)$ & $14(8.4)$ \\
\hline Septicemia, n (\%) & $12(7.5)$ \\
\hline Deep sternal wound infection, n (\%) & $0(0.0)$ \\
\hline ICU length of stay (days), median [IQR] & $3[1,6]$ \\
\hline Hospital length of stay (days), median [IQR] & $10[7,17]$ \\
\hline \multicolumn{2}{|l|}{ FET complications } \\
\hline \multicolumn{2}{|l|}{ Endoleaks } \\
\hline \multicolumn{2}{|l|}{ Type 1A endoleak } \\
\hline $\begin{array}{l}\text { Total arch replacement + FET with hybrid } \\
\text { arch graft (Thoraflex, Evita, Cook Hybrid } \\
\text { grafts), } \mathrm{n}(\%)\end{array}$ & $0 / 108(0.0)$ \\
\hline $\begin{array}{l}\text { Total arch replacement + FET with off-label } \\
\text { TEVAR grafts, } n(\%)\end{array}$ & $1 / 39(2.6)$ \\
\hline Hemiarch replacement + TEVAR, n (\%) & $5 / 20(25.0)$ \\
\hline Unplanned type 1B endoleak & $2(1.2)$ \\
\hline Type 2 endoleak & $12(7.2)$ \\
\hline Type 3 endoleak & $0(0.0)$ \\
\hline Type 4 endoleak & $1(0.6)$ \\
\hline New distal aortic dissection & $0(0.0)$ \\
\hline Distal aortic rupture & $0(0.0)$ \\
\hline Stent migration & $0(0.0)$ \\
\hline Stent graft-induced new entry & $1(0.6)$ \\
\hline
\end{tabular}

$\mathrm{SCI}$, spinal cord ischemia; ICU, intensive care unit; IQR, interquartile range; FET, frozen elephant trunk; TEVAR, thoracic endovascular aortic repair. in our series: (I) hybrid arch graft; (II) hemiarch with antegrade TEVAR deployment. First, the manufactured hybrid arch grafts (Thoraflex, Evita, and Cook Hybrid graft) are single-piece hybrid prostheses that are designed specifically for arch repair through a sternotomy with antegrade deployment under circulatory arrest. These hybrid grafts incorporate a surgically sewn proximal anastomosis in the arch, thus effectively eliminating the risk of type 1A endoleak around the stent graft, consistent with our experience where no proximal endoleaks were observed. The Thoraflex and Evita grafts are designed to conform to the curvature of the arch whereas the Cook Hybrid graft incorporates an uncovered polyester cuff at the proximal end of a standard Cook Alpha thoracic stent graft. Of the three hybrid arch grafts, the nitinol-ringed stent of the Thoraflex device exerts the least amount of radial force, which may be advantageous in the setting of acute dissection but may lead to under expansion and kinking requiring post-deployment ballooning in chronic dissections. The hybrid arch grafts have the distinct advantage of being specifically designed for a FET procedure (6-9). They treat the entire arch, minimize the risk of proximal endoleaks and late reintervention via sternotomy, and provide a stable platform for distal endovascular or open intervention. The reconstruction of the supra-aortic vessels and the level of the distal anastomosis (zone 2 or 3 ) is variable and depends on patient anatomy and surgeon preference. Although the hybrid arch grafts provide definitive repair, they require a total arch repair with added complexity and time. These devices may not be easy for non-aortic surgeons, and those who are not facile with this technique in the elective setting are unlikely to offer it for repair of an acute type A dissection.

The technique of a hemiarch repair with antegrade deployment of a TEVAR graft for acute type A dissection gained acceptance after early reports demonstrating improved outcomes in patients with malperfusion $(10,11)$. The advantages of this approach are that it is technically simple, adds minimal risk to a hemiarch procedure, and can be readily applied by the non-specialized cardiac surgeon. The main disadvantage is that this technique does not consistently obliterate the false lumen in the arch and may not provide a stable platform for future endovascular interventions. Type $1 \mathrm{~A}$ endoleaks are common (25\% in this series) and may require re-sternotomy with a formal arch and FET procedure if the distal aorta becomes aneurysmal. Several modifications of this technique have been proposed to improve the false lumen obliteration across the arch, 
including more proximal placement of the stent graft with in-situ fenestration and direct branch vessel stenting of the left subclavian artery (12). Although this has increased the favorable remodeling across the arch, the main pitfall of this simplified FET technique remains the risk of proximal endoleak and late aneurysmal degeneration. It continues to be a useful tool in patients with acute DeBakey I dissection, particularly those with distal malperfusion who either do not require or are not eligible for a hybrid arch graft.

Stroke and SCI remain the Achilles' heel of aortic arch repair with the FET technique. In this series, patients had a substantial burden of preoperative cerebrovascular disease (19\%) and over a third were undergoing urgent or emergent surgery, contributing to the relatively high observed rates of stroke and SCI. Nearly all patients received ACP with at least moderate systemic hypothermia, however, we did not collect the exact conduct of the ACP including flow and pressure rates. Prospective studies on arch repair should collect such details and would ideally adjudicate neurological endpoints with formal neurological assessment and magnetic resonance imaging (MRI) imaging of the brain. Despite improvements in surgical technique, graft selection, and patient management, the risk of SCI remains an important consideration for patients undergoing FET repair. In a recent meta-analysis of twelve studies including 1,803 patients, FET was associated with a significant increase in SCI compared to arch repair with a conventional Dacron elephant trunk (OR: 2.20, P=0.02) (3). Although we did not find a correlation between FET length and risk of SCI, we did not report the depth of FET implantation on computed tomography (CT), which has been previously reported as an important risk for SCI. The use of spinal drains as adjuncts to FET procedures has been the subject of debate and has a variable practice among aortic surgeons. We used prophylactic drains liberally in the setting of chronic dissection and when longer FET grafts were used.

The main limitation of this study is its retrospective nature and relatively small sample size. The study was not sufficiently powered to detect differences between surgical approaches and treatment of different aortic pathologies. Circulatory arrest temperature measurements were not standardized across all centers, with the majority of centers using nasopharyngeal measurements and others also using bladder measurements. Stroke was diagnosed clinically and on CT/MRI imaging and was not systematically screened for by a neurologist. Furthermore, the outcomes were limited to early in-hospital clinical and imaging outcomes. Nevertheless, the study reports the outcomes of FET repair with a wide variety of techniques and from several different centers, thus increasing the generalizability of the findings.

In conclusion, we have reported the contemporary experience with FET repair in 167 patients across six CTAC sites. This experience demonstrated good early clinical outcomes using a wide variety of approaches in patients with both aneurysmal disease and aortic dissections in the elective and urgent setting. Further investigation of the various devices with long-term imaging endpoints will be important to establish each technique within the armamentarium of hybrid aortic arch repair with FET.

\section{Acknowledgments}

None.

\section{Footnote}

Conflicts of Interest: MWAC has received speaker's honoraria from Medtronic, Edwards Lifesciences, Terumo Aortic, Boston Scientific, Abbott Vascular. MO has consultancy agreements with Gore, Ascyrus Medical, and Medtronic Inc. The other authors have no conflicts of interest to declare.

Open Access Statement: This is an Open Access article distributed in accordance with the Creative Commons Attribution-NonCommercial-NoDerivs 4.0 International License (CC BY-NC-ND 4.0), which permits the noncommercial replication and distribution of the article with the strict proviso that no changes or edits are made and the original work is properly cited (including links to both the formal publication through the relevant DOI and the license). See: https://creativecommons.org/licenses/by-nc-nd/4.0/.

\section{References}

1. Tian DH, Wan B, Di Eusanio M, et al. A systematic review and meta-analysis on the safety and efficacy of the frozen elephant trunk technique in aortic arch surgery. Ann Cardiothorac Surg 2013;2:581-91.

2. Shrestha M, Bachet J, Bavaria J, et al. Current status and recommendations for use of the frozen elephant trunk technique: a position paper by the Vascular Domain of EACTS. Eur J Cardiothorac Surg 2015;47:759-69.

3. Hanif H, Dubois L, Ouzounian M, et al. Aortic arch reconstructive surgery with conventional techniques vs frozen elephant trunk: a systematic review and meta- 
analysis. Can J Cardiol 2018;34:262-73.

4. Bozso SJ, White A, Nagendran J, et al. Hybrid aortic arch and frozen elephant trunk reconstruction: bridging the gap between conventional and total endovascular arch repair. Expert Rev Cardiovasc Ther 2018;16:209-17.

5. Hage A, Ginty O, Power A, et al. Management of the difficult left subclavian artery during aortic arch repair. Ann Cardiothorac Surg 2018;7:414-21.

6. Chu MWA, Losenno KL, Dubois LA, et al. Early clinical outcomes of hybrid arch frozen elephant trunk repair with the thoraflex hybrid graft. Ann Thorac Surg 2019;107:47-53.

7. Shrestha M, Martens A, Kaufeld T, et al. Single-centre experience with the frozen elephant trunk technique in 251 patients over 15 years. Eur J Cardiothorac Surg 2017;52:858-66.

8. Leontyev S, Tsagakis K, Pacini D, et al. Impact of clinical factors and surgical techniques on early outcome of patients treated with frozen elephant trunk technique by

Cite this article as: Ouzounian M, Hage A, Chung J, Stevens LM, El-Hamamsy I, Chauvette V, Dagenais F, Cartier A, Peterson M, Harrington A, Boodhwani M, Guo M, Bozinovski J, Fox S, Guo L, Chu MWA; on behalf of the Canadian Thoracic Aortic Collaborative. Hybrid arch frozen elephant trunk repair: evidence from the Canadian Thoracic Aortic Collaborative. Ann Cardiothorac Surg 2020;9(3):189-196. doi: 10.21037/acs2020-fet-22 using EVITA open stent-graft: results of a multicentre study. Eur J Cardiothorac Surg 2016;49:660-6.

9. Shrestha M, Kaufeld T, Beckmann E, et al. Total aortic arch replacement with a novel 4-branched frozen elephant trunk prosthesis: single-center results of the first 100 patients. J Thorac Cardiovasc Surg 2016;152:148-59.e1.

10. Pochettino A, Brinkman WT, Moeller P, et al. Antegrade thoracic stent grafting during repair of acute DeBakey I dissection prevents development of thoracoabdominal aortic aneurysms. Ann Thorac Surg 2009;88:482-9; discussion 489-90.

11. Preventza O, Cervera R, Cooley DA, et al. Acute type I aortic dissection: traditional versus hybrid repair with antegrade stent delivery to the descending thoracic aorta. J Thorac Cardiovasc Surg 2014;148:119-25.

12. Roselli EE, Idrees JJ, Bakaeen FG, et al. Evolution of simplified frozen elephant trunk repair for acute DeBakey type i dissection: midterm outcomes. Ann Thorac Surg 2018;105:749-55. 